\title{
Transport in quantum dot stacks using the Transfer Hamilto- nian method in self-consistent field regime
}

\author{
S. Illera ${ }^{(a)}$, J. D. Prades, A. Cirera and A. Cornet \\ MIND/IN² UB Departament d'Electrònica, Universitat de Barcelona, C/Martí i Franquès 1, E-08028 Barcelona, \\ Spain
}

PACS 72.10.Bg - General formulation of transport theory

PACS 73.63.-b - Electronic transport in nanoscale materials and structures

PACS 73.63.Kv - Quantum dots

\begin{abstract}
The non-coherent rate equation approach to the electrical transport in a serial quantum dot system is presented. The charge density in each quantum dot is obtained using the transfer Hamiltonian formalism for the current expressions. The interaction between the quantum dots and quantum dot and the electrodes are introduced by transition rates and capacitive couplings. Within this framework analytical expressions for the current and the charge in each quantum dot are presented. The effects of the local potential are computed within the selfconsistent field regime.

Despite the simplicity of the model, well-known effects are satisfactorily explained and reproduced. We also show how this approach can be extended into a more general case.
\end{abstract}

Introduction. - Confined structures have been available to the experimentalist for a very long time, the MOS (metal-oxide-semiconductor) transistor is the archetype of a confined two dimensional system [1]. Nevertheless, the possibility to enhance this confinement by embedding low-dimensional structures in an insulating matrix has renewed the interest. These structures (quantum dots, wires or layers) can be used in single electron device [2], new memory concepts [3] and photo or electroluminescent devices [4].

Due to the fabrication processes, all these structures are created in a multi layer structure. Therefore, the final structure intrinsically is a superlattice of insulatorsemiconductor bilayers. In this configuration, transport occurs in series, from one layer to the next one [5]. In the case of quantum dots (Qds), this makes the serial transport between Qd the most relevant case of study. Concerning single electron devices, they are currently conceived to take advantage of tunnel current between quantum states belonging to nano-scale particles $[6,7]$. Until now, research has been mostly concentrate on single quantum dots, but rapid progress in mi-

(a) E-mail: sillera@el.ub.es crofabrication tecnology has made possible the extension to couple of quantum dots system with aligned levels [8-10]. These simple systems have been studied using many body approaches including non-equilibrium Green's function formalism [11-13]. Up to now the only computation of transport in an extended arbitrary array of quantum dots was done by Carreras et al [14] but no local potential due to selfcharge was included. In this work, we use non-coherent rate equations to study the electrical transport in QDs [15-17]. Electron transport and charge densities inside the Qds depend on the tunnel transparency of the barriers limiting each dot. Thus, the interaction between the Qds, and between Qds and the electrodes are introduced by transition rates and capacitive couplings. In order to effectively solve the multielectron problem, the effects of the local potential are computed within the selfconsistent field (SCF) regime. As an example of the validity of the here presented methodology, we studied the total current and charge distribution for two Qds dots in a serial configuration.

Some well-known experimental results, such as negative differential resistance (NDR) and resonant con- 
ductance $[18,19]$, are satisfactorily reproduced. We also show how this approach can be extended to large chains of QDs.

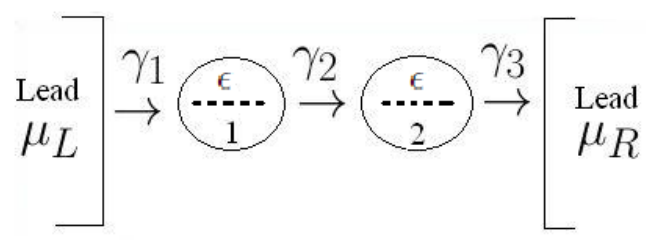

Figure 1: Schematic view of the system under study. Both Qds are the intermediate transport region in contact with a left and right leads. The leads are defined by the electrochemical potential $\left(\mu_{R}\right.$ and $\left.\mu_{L}\right)$. Only one state is considered in each Qd and it is coupled with its first neighbor. These couplings are described by the transition rates $\gamma_{i}$.

Theoretical background. - Let us consider two leads (contacts) coupled to an intermediate transport region with no voltage applied. In these conditions, the electrochemical potential is common and the system is in equilibrium. For the sake of clarity, this situation can be picture, for example, as a quantum dot (Qd) inside of an insulating matrix located between the two electrodes. In this equilibrium state, the average number of electrons in any energy level is given by the Fermi Dirac distribution function. If an external bias voltage $(V)$ is applied, it will drive the system out of equilibrium changing the electrochemical potential of the leads $\mu_{L}-\mu_{R}=q V$, where $\mu_{L}$ and $\mu_{R}$ are the electrochemical potential in the left and right lead, creating electron currents as a consequence of the different population in each lead. Initially, in the intermediate transport region $(\mathrm{Qd})$, we only consider one state with energy level $\epsilon$ that lies between the electrochemical potentials of the two contacts. The contacts see $f_{L}(\epsilon)$ and $f_{R}(\epsilon)$ electrons occupying the single state where $f_{L}$ and $f_{R}$ are the Fermi Dirac distribution function of the left and right contact respectively. Thus, the flux $\left(I_{L}\right)$ between the left contact and the intermediate region is $[20]$

$$
I_{L}=I_{L}^{+}-I_{L}^{-}=q \frac{\gamma_{L}}{\hbar}\left(f_{L}-N\right)
$$

where $I_{L}^{+}$is the incoming flux from contact to the region and $I_{L}^{-}$is the flux from the region to contact. $\gamma_{L} / \hbar$ is the transition rate and $\mathrm{N}$ is the average number of electrons in the energy level. The previous expression refers the net flux between the incoming flow from the left lead to the state and the outflow from the state to the left lead. Similarly the net flux across the right junction $\left(I_{R}\right)$ can be written as

$$
I_{R}=q \frac{\gamma_{R}}{\hbar}\left(f_{R}-N\right)
$$

In order to find $\mathrm{N}$ we can write a rate equation for the system. The rate equation calculation considers the dynamic behavior of the number of electrons in the system balanced with the incoming and outcoming flux [21]. Using the previous expressions for the currents the rate equation is

$$
\frac{d N}{d t}=I_{L}+I_{R}
$$

. By solving it in the steady state we obtain

$$
N=\frac{\gamma_{R} f_{R}+\gamma_{L} f_{L}}{\gamma_{R}+\gamma_{L}}
$$

- Once we have the expression for $\mathrm{N}$ we can derivate the steady state current as:

$$
I=\frac{q}{\hbar} \frac{\gamma_{L} \gamma_{R}}{\gamma_{L}+\gamma_{R}}\left(f_{L}-f_{R}\right)
$$

This result illustrates that there is only current flowing from lead to lead when the electrochemical potentials of the leads are different, $\mu_{R} \neq \mu_{L}$, therefore $f_{L}(\epsilon) \neq f_{R}(\epsilon)$. Also, in order to have transport, the energy level $\epsilon$ must lie between the electrochemical potentials of the leads $\left(\mu_{R}\right.$ and $\left.\mu_{L}\right)$. Once the energy level is located between the electrochemical potentials of the leads the average charge in the level will be time dependent until a steady state is reached. This is, when the currents in and out of the energy level are equal $[15,16]$.

Let's extend now the previous formalism to the system represented in fig. (1). We consider that each Qd is only coupled with its first neighbor, either another Qd or a lead. The general expression for the current between two parts of the system is

$$
I_{i, j}=q \frac{\gamma_{i, j}}{\hbar}\left(f_{i}-f_{j}\right)
$$

where the subscripts $i, j$ refer to the parts involved in the transport, $\gamma_{i, j} / \hbar$ is the transition rate and $f_{i}, f_{j}$ are the distribution functions of the different parts. The only known distribution function is the one that corresponds to the electrodes, which is the Fermi Dirac function. Using the rate equation, we can write a system of equations (one per $\mathrm{Qd}$ ) in order to find the average number of electrons in each Qd

$$
\begin{aligned}
& \frac{d N_{1}}{d t}=\frac{q}{\hbar} \gamma_{1}\left(f_{L}-N_{1}\right)+\frac{q}{\hbar} \gamma_{2}\left(N_{2}-N_{1}\right) \\
& \frac{d N_{2}}{d t}=\frac{q}{\hbar} \gamma_{3}\left(f_{R}-N_{2}\right)+\frac{q}{\hbar} \gamma_{2}\left(N_{1}-N_{2}\right)
\end{aligned}
$$

where the $\gamma_{i}$ coefficients are the transition rates described in fig. (1). $f_{L}$ and $f_{R}$ are the Fermi Dirac 
distributions (evaluated at $\epsilon$ ) and $N_{i}$ is the average number of electrons in the $i^{\text {th }} \mathrm{Qd}$. The steady solution of eq. (7) and eq. (8) is

$$
\begin{aligned}
& N_{1}=\frac{\gamma_{1}\left(\gamma_{2}+\gamma_{3}\right) f_{L}+\gamma_{2} \gamma_{3} f_{R}}{\left(\gamma_{1}+\gamma_{2}\right)\left(\gamma_{2}+\gamma_{3}\right)-\gamma_{2}^{2}} \\
& N_{2}=\frac{\gamma_{3}\left(\gamma_{2}+\gamma_{1}\right) f_{R}+\gamma_{2} \gamma_{1} f_{L}}{\left(\gamma_{1}+\gamma_{2}\right)\left(\gamma_{2}+\gamma_{3}\right)-\gamma_{2}^{2}}
\end{aligned}
$$

Besides, the outcoming net flux from the last Qd to the right lead is

$$
I=q \frac{\gamma_{3}}{\hbar}\left(f_{R}-N_{2}\right)
$$

This result helps us to consider a $\mathrm{N}$-independent way to express the current using eq. (10)

$$
I=\frac{q}{\hbar} \frac{\gamma_{1} \gamma_{2} \gamma_{3}}{\gamma_{1} \gamma_{2}+\gamma_{1} \gamma_{3}+\gamma_{2} \gamma_{3}}\left(f_{L}-f_{R}\right)
$$

From the physical point of view, this expression can be interpreted as follows: the three barriers in the $\mathrm{Qd}$ stack act as a series connection of resistors $R_{\text {total }}=$ $\sum_{i}^{N} R_{i}$. Where $R_{i}$ is the inverse of transition rates.

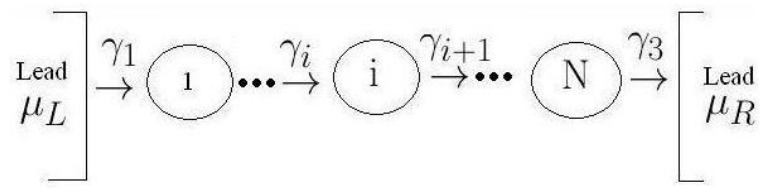

Figure 2: Extended case to a $N$ chain of Qds. All the elements only interact with their firsts neighbors with a transition rate $\gamma_{i}$.

It is straightforward to extend the model presented before to the general case that is shown in fig. (2). Writing a rate equation for each $\mathrm{Qd}$ and computing the net fluxes from its neighbors, we can evaluate the average number of electrons in each Qd in the steady state:

$$
N_{i}=\frac{\gamma_{i} N_{i-1}+\gamma_{i+1} N_{i+1}}{\gamma_{i}+\gamma_{i+1}} ; N_{0}=f_{L} ; N_{N+1}=f_{R}
$$

Therefore the expression for the current can be obtained as a function of the Fermi Dirac distribution function of the leads. In a general case, the transport condition in these configurations can be summarized as

$$
\mu_{L}>\epsilon_{1}=\ldots=\epsilon_{i}=\ldots=\epsilon_{N}>\mu_{R}
$$

where $\epsilon_{i}$ is the energy level of the intermediate region states and $\mu_{L}$ and $\mu_{R}$ are the electrochemical potential of the leads. This restrictive condition is relaxed by the inclusion of the energy level broadening.

The standard way to introduce the broadening of the energy levels as a consequence of the contacts is

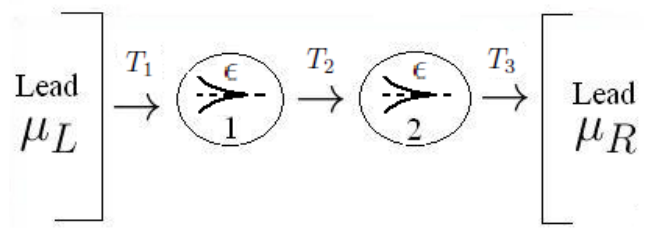

Figure 3: Representation of the system under study. Two Qds with an energy level broadened by the coupling between the different parts. The different transmission probabilities between the elements are shown.

to assign a Lorentzian shape to the density of states $[22-24]$

$$
\rho_{i}(E)=\frac{\frac{\delta}{2 \pi}}{(E-\epsilon)^{2}+\left(\frac{\delta}{2}\right)^{2}}
$$

$\rho_{i}$ is the density of states (DOS) of the $i^{\text {th }} \mathrm{Qd}$ and $\epsilon$ is the initial single energy level in each Qd. The broadening $\delta$ is proportional to the strength of the coupling between the $\mathrm{Qd}$ and the sorrounding elements, $\delta=\sum_{i} \gamma_{i}$, where $\gamma_{i}$ are the different transition rates introduced before and the sum only runs over the possible transitions. Since the transition rates can be different, the broadening of each Qd DOS depends on the different couplings. This effect changes the current expressions since we need to take into account the current trough the multiple energy states. Using the transfer Hamiltonian formalism assuming no inelastic scattering $[25,26]$, the current between two parts of the system can be written as

$$
I_{i j}^{-}=\frac{4 \pi q}{\hbar} \int T_{i j}[E] \rho_{i}(E) f_{i}(E) \rho_{j}(E)\left(1-f_{j}(E)\right) d E
$$

for the outcomming flux, and for the incoming flux

$$
I_{i j}^{+}=\frac{4 \pi q}{\hbar} \int T_{j i}[E] \rho_{j}(E) f_{j}(E) \rho_{i}(E)\left(1-f_{i}(E)\right) d E
$$

being the net current flux

$$
\begin{aligned}
I_{i j} & =I_{i j}^{+}-I_{i j}^{-}= \\
& =\frac{4 \pi q}{\hbar} \int T_{i j}[E] \rho_{i}(E) \rho_{j}(E)\left(f_{j}(E)-f_{i}(E)\right) d E
\end{aligned}
$$

where $T_{i j}(E)$ is the transmission probability, $\rho_{i}(E)$ and $\rho_{j}(E)$ are the density of states meanwhile $f_{i}(E)$ and $f_{j}(E)$ are the distribution functions of the different parts of the system $\left(f_{L}\right.$ and $f_{R}$ for leads and $n_{i}$ for $\left.i^{\text {th }} \mathrm{Qd}\right)$. The previous expressions show transport processes in which an electron in one side $\left(\rho_{i}(E) f_{i}(E)\right.$ occupied states) goes through a barrier into an available state in the other side $\left(\rho_{j}(E)\left(1-f_{j}(E)\right)\right.$ with a probability $T_{i j}(E)$. We can also assume symmetry in the transmission coefficient: $T_{i j}=T_{j i}[27]$ and obtain the 
current expression eq. (18). Now the total charge $N_{i}$ inside the $i^{\text {th }} \mathrm{Qd}$ is

$$
N_{i}=\int \rho_{i}(E) n_{i}(E) d E
$$

where the distribution function $n_{i}(E)$ can be found using the previous rate equation formalism for each energy step. Now $n_{i}(E)$ acts as a continuous nonequilibrium distribution function for a given energy.

Applying the rate equation with the new expressions of the currents, where the DOS and the transmission probability appear explicitly, the distribution functions in each Qd for the system represented in fig. (3) are

$$
\begin{aligned}
\frac{d n_{1}}{d t} & =\frac{4 \pi q}{\hbar}\left(T_{1} \rho_{L} f_{L}+T_{2} \rho_{2} n_{2}-n_{1}\left(T_{1} \rho_{L}+T_{2} \rho_{2}\right)\right) \\
\frac{d n_{2}}{d t} & =\frac{4 \pi q}{\hbar}\left(T_{3} \rho_{R} f_{R}+T_{2} \rho_{1} n_{1}-n_{2}\left(T_{2} \rho_{1}+T_{3} \rho_{R}\right)\right)
\end{aligned}
$$

and the expression for the current is (in comparation with eq. (12))

$I=\frac{4 \pi q}{\hbar} \int \frac{T_{1} T_{2} T_{3} \rho_{L} \rho_{1} \rho_{2} \rho_{R}}{T_{1} T_{2} \rho_{L} \rho_{1}+T_{1} T_{3} \rho_{R} \rho_{L}+T_{2} T_{3} \rho_{2} \rho_{R}}\left(f_{L}-f_{R}\right)$

where $\rho_{L}$ and $\rho_{R}$ are the DOS of the leads and $\rho_{1}$ and $\rho_{2}$ are the DOS in each Qd. As in the case of $\mathrm{N}$-independent current expressions the condition $f_{L} \neq f_{R}$ must be fulfilled. In order to have transport the energy levels, now the DOS, must lie between the electrochemical potentials of the leads and overlapping of the QD DOS is necessary (14). This means that the electron needs available states in each part of the system in order to transport from left lead to the right lead trough the Qd system.

Another factor that we need to consider is how the voltage applied to the external electrodes change the electrostatic potential inside each Qd. It is expected that this can play an important role to determining the shape of the current-voltage characteristics. The classical solution for the potential at each quantum dot $\left(V_{i}\right)$ involves the Poisson equation

$$
\vec{\nabla} \cdot\left(\epsilon_{r} \vec{\nabla} V_{i}\right)=-\frac{q \triangle N}{\Omega \epsilon_{0}}
$$

where $\epsilon_{r}$ is the relative permittivity of the dielectric media, $\epsilon_{0}$ is the vacuum permittivity and $\Omega$ is the $\mathrm{Qd}$ volume. The general solution for the potential energy $U_{i}=-q V_{i}$ in the $i^{\text {th }} \mathrm{Qd}$ is [20]

$$
U_{i}=U_{L, i}+\frac{q^{2}}{C_{t o t, i}} \triangle N_{i}
$$

being $U_{L, i}$ the Laplace solution of the system and $\triangle N_{i}$ the change in the number of electrons, calculated respect to the reference number $N_{0}$ originally in the $i^{t h}$
Qd. The charge energy constant $U_{0, i}=q^{2} / C_{t o t, i}$ is the potential increase as a consequence of the electron addition. Considering capacitive coupling and suitable boundary conditions, the Laplace solution is [14]

$$
U_{L, i}=\sum_{j} \frac{C_{i, j}}{C_{t o t, i}}\left(-q V_{j}\right)
$$

where $C_{i, j}$ is the capacitive coupling between the different parts of the system. The subscript $j$ runs over all the parts of the system meanwhile $i$ only runs over the $N$ Qds, and $C_{t o t, i}=\sum_{j} C_{i, j}$. The effects of local potential on each Qd should be computed in the Qd DOS $\rho_{i}(E) \rightarrow \rho_{i}\left(E-U_{i}\right)$ which modify the Qd charge and the currents. In eq. (24) we observe that the local potential depends on the increasing charge density but at the same time the charge depends on the DOS that it is modified by the local potential. These considerations impose a self-consistent solution of eq. (19) and eq. (24).

$d E$

Results. - For simplicity, we consider that the transmission probability is the same in all the system and, because of the small width of the DOS inside of each Qd, we also consider constant transmission probabilities $T_{1}=T_{2}=T_{3}$. In addition to this, we assume that the QD are identical. Therefore, the energy level is the same with a value $\epsilon$. We do not consider direct transmission between the leads. Besides, due to the position of each Qd in fig. (3) we contemplate that the first $\mathrm{Qd}$ is capacitively coupled with the left lead and its neighbor Qd while the second Qd is coupled with the first Qd and the right lead. Thus the Laplace solution result for each Qd is

$$
\begin{gathered}
U_{L, 1}=\frac{C_{c}}{C_{t o t}^{\prime}} U_{2} \\
U_{L, 2}=\frac{C_{s}}{C_{t o t}}(-q V)+\frac{C_{c}}{C_{t o t}} U_{1}
\end{gathered}
$$

where $\mathrm{V}$ is the bias voltage applied to right lead, whilst the left lead remains at zero. $C_{s}$ is the capacity coupling between the second $\mathrm{Qd}$ with the right lead while $C_{s}^{\prime}$ is the coupling between the first Qd with the left lead. $C_{c}$ is the capacity between Qds. So, we define the total capacitive coupling of each Qd as $C_{t o t}^{\prime}=C_{s}^{\prime}+C_{c}$ and $C_{t o t}=C_{s}+C_{c}$ for the first and second Qd respectively. Figure (4) shows current voltage curves obtained for the system defined in fig. (3) with different $C_{s} / C_{c}$ ratios rangin from zero to one while the corresponding conductance is depicted in fig. (5). The I(V) curve is strongly dependent of $C_{s}$ and $C_{c}$ trough the Laplace solution of the system. The value of the capacity indicates how the system is coupled. When $C_{s}$ 

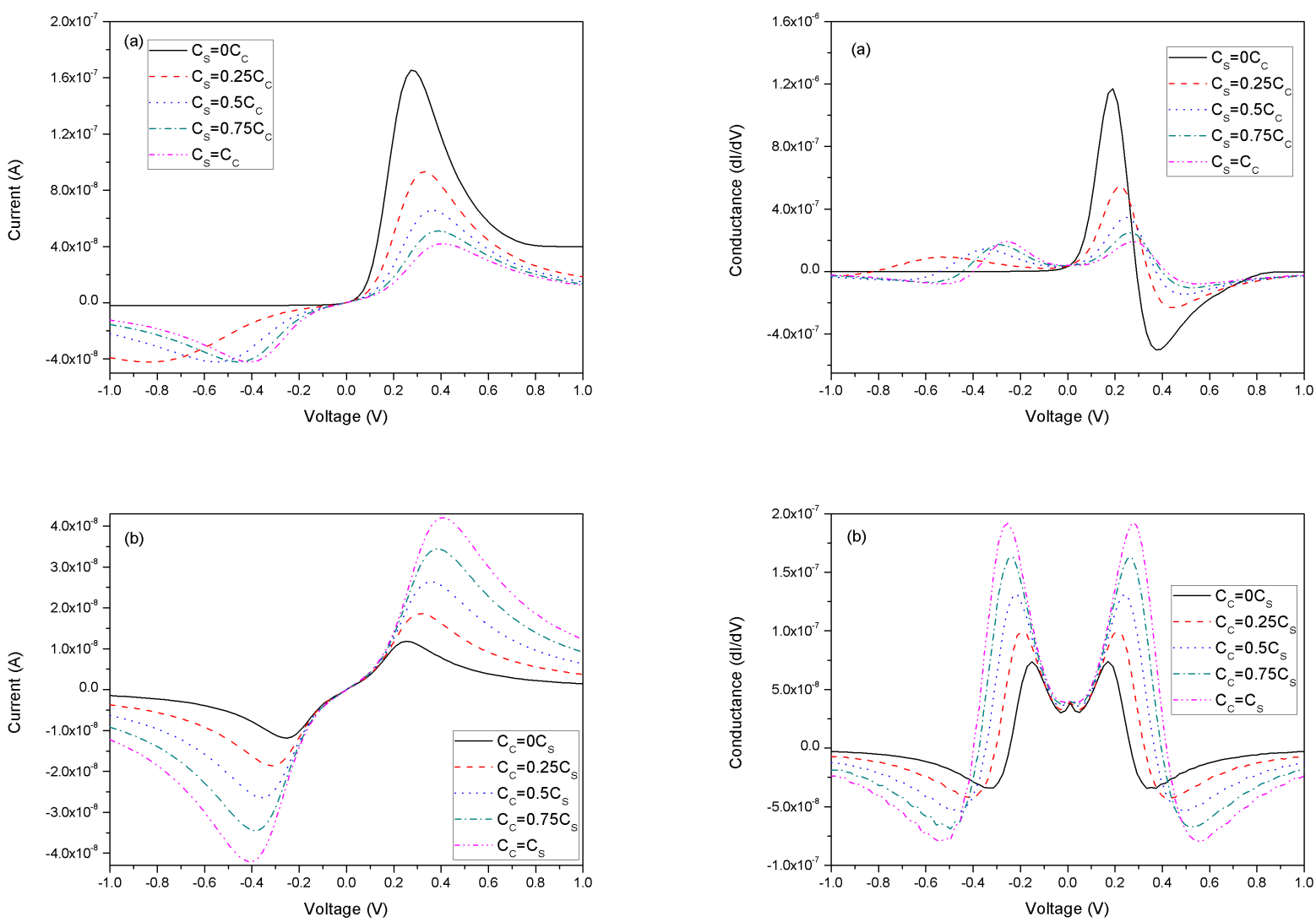

Figure 4: Current voltage curves obtained for fig. (3). (4-a) as a function of the lead-Qd capacity. Showing a rectifying effect when the system is weakly coupled with the leads. The condition expressed in (14) can not be fulfilled hence no current appears.4-b) Resonant current peak is obtained due to the different electrostatic coupling of the Qds. When the voltage increases the separation between the energy levels increases and the overlapping between the DOS decrease therefore NDR appears. The simulation parameters are: $\mu_{L}=0, \mu_{R}=-q V, T_{1}=T_{2}=T_{3}=0.005, k_{\beta} T=0.025 \mathrm{eV}$, $U_{0}=0.25 \mathrm{eV}, \epsilon=0.2 \mathrm{eV}$ and $\epsilon_{r} \epsilon_{0}=1$.

tends to zero, the two Qds are electrically decoupled with the leads; this case implies a weakly coupled system. The other limit case is when the value of the capacity between $\mathrm{Qd}\left(C_{c}\right)$ goes to zero which means no electrical influence between Qds. The Poisson term follows the charge $N_{i}$ in each Qd therefore it is always positive inducing a shift on the potential. In order to explain the $\mathrm{I}(\mathrm{V})$ curves, we studied the evolution of the energy level of each Qd as a function of the applied bias voltage taking into account only the Laplace term. The equation for the first $\mathrm{Qd}$ is

$$
\epsilon_{1}(V)=\epsilon+\frac{C_{c}}{C_{t o t}^{\prime}} U_{2}
$$

Figure 5: Conductance characteristics obtained in the same system as before as a function of the capacitive coupling between the Qds and the lead (a) and between Qds (b). Rectifying effect, resonant peaks and NDR are obtained.

and for the second one it is

$$
\epsilon_{2}(V)=\epsilon+\frac{C_{s}}{C_{t o t}}(-q V)+\frac{C_{c}}{C_{t o t}} U_{1}
$$

. This is a set of recursive equations, eq. (26) and eq. (27). In order to analyze the results as a function of the applied bias voltage we used the relation $a+a r+$ $a r^{2} \ldots=a /(1-r)$ for a geometric series. Finally, we obtained

$$
\begin{gathered}
\epsilon_{1}=\epsilon+\frac{C_{c} C_{s}}{C_{t o t}^{\prime} C_{t o t}-C_{c}^{2}}(-q V) \\
\epsilon_{2}=\epsilon+\frac{C_{s}}{C_{t o t}-C_{c}^{2} / C_{t o t}^{\prime}}(-q V)
\end{gathered}
$$

As we can see from the previous expressions $\epsilon_{1}$ increases faster than $\epsilon_{2}$. Thus, the transport condition (14) can not be accomplished. Introducing the DOS broadening relaxes this restrictive condition and electron transport takes place as a result of the energy channel overlapping. When the voltage increases 
the separation between $\epsilon_{1}$ and $\epsilon_{2}$ increases therefore the overlapping between the Qd DOS decrease closing some channels and decreasing the current trough the system, as a consequence, a negative differential resistance (NDR) appears. [28] The I(V) peak is related to the maximum overlapping between the Qd DOS. The width of the peak is related to the DOS broadening. On the other hand, if the system is decoupled from the right lead $\left(C_{s}=0\right)$, the position of the energy level becomes independent on the applied bias voltage. Therefore transport only occurs in one direction, producing a rectifying effect. In the opposite direction the current would be constant. Nevertheless, in fig. (4) we do not observe this behavior because the first Qd increase its charge. Therefore, the charge change dominates the Poisson solution while the charge in the second Qd is not the same creating different potentials in each Qd thus changing the energy level position. This configuration is a particular case that eq. (30) and eq. (31) do not consider because they are a first approximations.

Conclusions. - In conclusion, a simple phenomenological model of the conduction between quantum dots has been developed based on rate equations for non-coherent Qd system. This approach provides a simple and transparent method to describe the electron transport. First, we consider a system of two Qd linked by a ballistic channel and connected with the emitter and collector reservoirs. Level broadening has been introduced in the DOS changing the expression for current and the population inside each Qd. Despite its simplicity the effect of self-charge has been taken into account, by solving the Poisson equation with appropriate boundary conditions for each Qd. As we may expect the calculation of the local potential inside each $\mathrm{Qd}$ is the most important point. This local potential dominates the current characteristics since overlapping of the DOS is imposed in order to have electron transport. $\mathrm{I}(\mathrm{V})$ and conductance curves have been obtained in a range of capacity values. NDR and $\mathrm{I}(\mathrm{V})$ resonant peak have been observed. We also showed how this model can be extended easily to a large Qd chain.

This work was supported by NASCENT FP7-NMP245977 European project. A. Cirera acknowledges support from ICREA academia program. The authors thankfully acknowledge the Barcelona Supercomputing Center - Centro Nacional de Supercomputación.

\section{References}

[1] Ando T., Fowler A. B. and Stern F., Rev. Mod. Phys., 54 (1982) 437.
[2] Meirav U. and Foxman E. B., Semicond. Sci. Technol., 10 (1995) 255.

[3] Tiwari S., Rana F., Chan K., Shi L. and Hanafi H., Appl. Phys. Lett, 69 (1996) 1232.

[4] Lockwood D. J., Semiconductor and Semimetals, sect. Light Emission in Silicon From Physics to Devices, Vol. 49 (Academic Press, San Diego) 1998, p. 1-36

[5] Marcello Rosini, Carlo Jacoboni and Stefano Ossicini, Phys. Rev. B, 66 (2002) 155332.

[6] Ashoori R. C., Nature, 379 (1996) 413-419

[7] Cobden D. H., Bockrath M., McEuen P.L., RinZler A.G. and Smalley R. E., Phys. Rev. Lett, 81 (1998) 681.

[8] Haug R. J., Hong J. M. and Lee K. Y., Surf. Sci, 263 (1992) 415.

[9] VAn der VaART N. C., Godijn S. F. and Nazarov Y. V., Phys. Rev. Lett, 74 (1995) 4702-4705.

[10] Waugh F. R., Berry M. J., Mar D. J., Westervelt R. M., Vampman K. L. and Gossard A. C., Phys. Rev. Lett, 75 (1995) 4702.

[11] Levy Yeyati A., Martin-Rodero A. and Flores F., Phys. Rev. Lett, 71 (1993) 2991.

[12] Meir Y. and Wingreen N. S., Phys. Rev. Lett, 68 (1992) 2512.

[13] Meir Y., Wingreen N. S. and Lee P. A., Phys. Rev. Lett, 66 (1991) 3048.

[14] Carreras J., Jambois O., Lombardo S. and GarRIDO B., Nanotechnology, 20 (2009) 155201.

[15] Averin D. V., Korotkov A. N. and Likharev K., Phys. Rev. B, 44 (1991) 6199.

[16] Van Delft J. and Ralph D. C., Phys. Rep., 345 (2001) 61

[17] Gurvitz S. A., Phys. Rev. B, 57 (1998) 11.

[18] Borgstrom M., Bryllert T., Sass T., Gustafson B., Wernersson L. E., Seifert W. and Samuelson L., Appl. Phys. Lett., 78 (2001) 3232

[19] Bryllert T., Borgstrom M., Wernersson L. E., Seifert W. and Samuelson L., Appl. Phys. Lett., 82 (2003) 2655

[20] Datta S., Nanotechnology, 15 (2004) S433-S451.

[21] Niquet Y. M., Delerue C., Allan G. and Lannoo M., Phys. Rev. B, 65 (2002) 165334.

[22] Foxman E. B., Meirav U. et al, Phys. Rev. B, 50 (1994) 14193-14199.

[23] Massimiliano Di Ventra, Transport in Nanoscale Systems (Cambridge University Press) 2008

[24] Batra I. P., Solid State Commun, 124 (2002) 463-7.

[25] Passoni M. and Bottani C.E., Phys. Rev. B, 76 (2007) 115404.

[26] Payne M. C., J. Phys. C: Solid State Phys, 19 (1986) 1145-1155.

[27] Supriyo DatTa, Electronic Transport in Mesoscopic Systems (Cambridge University Press) 1995, p. 88

[28] This effect is well explained using a linear approximation, taking into account only the Laplace solution of (24). 